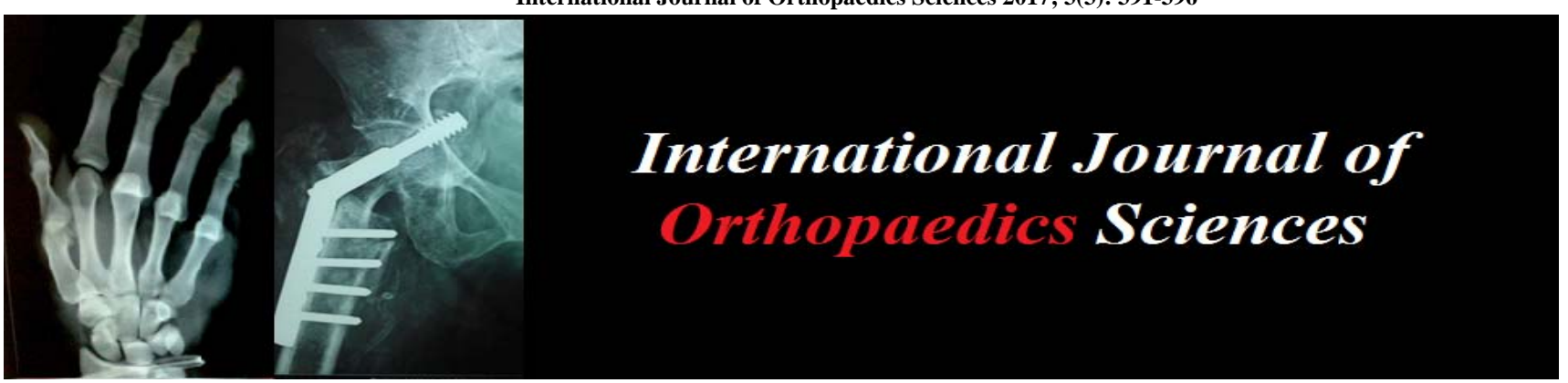

ISSN: 2395-1958

IJOS 2017; 3(3): 391-396

(C) 2017 IJOS

www.orthopaper.com

Received: 28-05-2017

Accepted: 29-06-2017

\section{R Benfayed}

Department of Orthopaedics and

Trauma Royal Infirmary of Edinburgh 51 Little France

Crescent Old Dalkeith Road Edinburgh EH16 4SA

\section{Moran}

Department of Orthopaedics and

Trauma Royal Infirmary of Edinburgh 51 Little France Crescent Old Dalkeith Road Edinburgh EH16 4SA

\section{AHRW Simpson}

Department of Orthopaedics and Trauma Royal Infirmary of Edinburgh 51 Little France Crescent Old Dalkeith Road Edinburgh EH16 4SA

\section{N Clement}

Department of Orthopaedics and Trauma Royal Infirmary of Edinburgh 51 Little France Crescent Old Dalkeith Road Edinburgh EH16 4SA

\section{Macdonald}

Department of Orthopaedics and

Trauma Royal Infirmary of Edinburgh 51 Little France Crescent Old Dalkeith Road Edinburgh EH16 4SA

\section{Correspondence}

\section{$R$ Benfayed}

Department of Orthopaedics and

Trauma Royal Infirmary of

Edinburgh 51 Little France

Crescent Old Dalkeith Road

Edinburgh EH16 4SA

\section{Retropatellar cartilage morphology and kneeling ability: cartilage wear and kneeling function of TKA patients}

\author{
R Benfayed, M Moran, AHRW Simpson, N Clement and D Macdonald
}

DOI: http://dx.doi.org/10.22271/ortho.2017.v3.i3f.68

\section{Abstract}

Objectives: To investigate the retro-patellar cartilage (RPC) morphology and the patterns of retropatellar cartilage wear as well as the patients' kneeling ability to determine whether the kneeling ability is related to the area and pattern of wear in the cartilage.

Methods: 30 patients undergoing primary TKAs participated in this study. The patients were seen in the preoperative assessment clinics and consented to allow intraoperative photographing of their retro patellar cartilage. These photographs were used to determine the area and pattern of wear by using Image $\mathrm{J}$ software. The total area of the retropatellar cartilage and the area of wear was measured and used to calculate the percentage of RPC wear. The percentages of retro patellar cartilage wear for all the patients were linked with their kneeling ability before the surgery.

Results: 15 patients (cases numbered 1-15, termed group A) were able to kneel, and 15 patients (cases numbered 16-30, termed group B) were unable to kneel. The \% of wears for all RPC images measured 3 times with a week interval and the average of the records in the 1st, 2nd, and 3rd week recorded for statistical analysis. Mean \% of RPC wears in-group A was (15\%), and in-group B was (36\%). Intra-Rater and Inter-Rater Reliability statistically assessed by using Intraclass Correlation Coefficient. Patients who able to kneel (Group A) had normal RPC superior surfaces with no damages in comparison with patients who unable to kneel (Group B) who had damaged superior surfaces medially and laterally. All the RPC surfaces (Superior, Middle and Inferior) were damaged in Group B patients (Unable to kneel).

Conclusion: No direct association was found between the retropatellar cartilage damage and the kneeling ability. The most damaged areas were found in the middle and lower thirds of retropatellar cartilage.

Keywords: Arthroplasty, Knee, Kneeling, Patella, Retropatellar Cartilage, Articular, Preoperative

\section{Introduction}

Most of the published studies have correlated the degree of RPC damage with patellofemoral pain but not with kneeling function (van der Heijden, Oei et al. 2016) ${ }^{[36]}$. Dye (2005) ${ }^{[9]}$ has said that increased cartilage wear leads to synovitis and that leads to pain. Different techniques have been used to assess the RPC damage; two cross-sectional studies used MRI to evaluate cartilage change in relation to particular types of occupational activities including knee bending. This study examined the changes in different compartments (Amin, Goggins et al. $2008{ }^{[1]}$, Teichtahl, Wluka et al. 2010) ${ }^{[34]}$. Horng et al. (2011) ${ }^{[17]}$ used a high-resolution 3DMRI to analyse regional deformation patterns as an indication of contact areas in patellar cartilage after different loading exercises. The articular surface of the patella is composed of thick hyaline cartilage, which provides an insensitive, avascular area specifically adapted to bearing high compressive loads (Rhee S.J, Haddad F.S, 2008) ${ }^{[29]}$. The patellar articular cartilage has to withstand huge forces and is the thickest hyaline cartilage in the body. The thickest point is $7 \mathrm{~mm}$ deep, and the facets of the patella are unique in shape and position to each knee (Kwak, Colman et al. 1997) ${ }^{[22]}$. The retro patellar cartilage is also unique, in that it does not follow the contours of the underlying subchondral bone, as is the norm elsewhere in the body (Stäubli, Dürrenmatt et al. 1999), patellar cartilage is more permeable and more compressive than cartilage elsewhere, (Froimson, Ratcliffe et al. 1997) ${ }^{[10]}$ which may, along with large contact pressures, and not following the contours of the bone explain why patella cartilage lesions are more prevalent than cartilaginous lesions elsewhere. 
An elaborate system of intraosseous and extraosseous vasculature provides blood supply to the patella (Björkström and Goldie $1980^{[3]}$, Kayler and Lyttle 1988) ${ }^{[21]}$. A peripatellar anastomotic ring consisting of six main arteries forms the extraosseous blood supply. These six arteries, supplying the ring, are (1) the articular branch of the descending genicular artery, (2) the medial superior genicular artery, (3) the medial inferior genicular artery, (4) the anterior tibial recurrent artery, (5) the lateral inferior genicular artery and (6) the descending branch of the lateral femoral circumflex artery. The superior part of the ring passes anterior to the quadriceps tendon, and the inferior portion passes posterior to the patellar tendon through the substance of the fat pad. The knowledge of PF contact points in different degrees of knee flexion and the biomechanics affecting the patterns and degrees of retro patellar cartilage wear are a useful basis for analysing the retro patellar cartilage morphology.

The patella articulates with the cartilage in the patellar groove on the distal femur between 10 and 100 degrees of flexion. The trochlea averages $31-34 \mathrm{~mm}$ in height (from the base of the ' $\mathrm{V}$ ' to the crest of the lateral condyle). However, there is considerable variation in trochlear height, width and shape within the population (Yoshioka, Siu et al. 1987) ${ }^{[37]}$. The patella is a shifting fulcrum of the extensor mechanism as the knee flexes and extends (Grelsamer, Proctor et al. 1994) ${ }^{[14]}$. The amount of contact the patella has with the femur varies depending on the position of the knee. The patella contact area is small and distal in early flexion, and increases in area and progresses proximally with increasing flexion (Hehne 1990) ${ }^{[16]}$. Maximum patella-femoral contact area occurs at 120 degrees of flexion (Huberti and Hayes 1984) ${ }^{[18]}$. The contact pressure between the patella and femur is high with the maximum pressure occurring at 90 degrees of flexion in knees with normal Q-angle (Huberti and Hayes 1984) ${ }^{[18]}$. During activities such as kneeling, the reactionary force through the patella-femoral joint is as much as 7.6 times the body weight. This pressure can increase significantly where the $\mathrm{Q}$ angle is increased, as in a valgus knee joint.

\section{Patients\& Methods}

30 patients undergoing primary TKAs (Triathlon- Single Radius implant design) were entered into this part of the study. These patients were recruited from six consultants' preoperative assessment clinics at RIE-NHS Lothian (November 2016 to February 2017). There were 20 women and 10 men, 16 underwent (Right) TKA and 14 (Left) TKA.

The patients were seen in the preoperative assessment clinics and consented to allow intraoperative photographing of their retro patellar cartilage. The process of intraoperative photographing standardized as follows:

Keeping the overhead lights away to avoid light reflection.

Marking of the patella- $\mathrm{S}=$ Superior, $\mathrm{I}=$ Inferior, $\mathrm{M}=$ Medial, $\mathrm{L}=$ Lateral.

The photo was taken after patella eversion and before nibbling of patella osteophytes.

The focal length between camera and knee standardised to the best photograph quality.

The photo was taken from the same side of the surgery.

The camera used -SONY Cyber-shot DSC-WX220B Compact Camera.

Photographs excluded from the study if they could not meet the process standards.

These images were used to determine the area and pattern of wear by using Image $\mathbf{J}$ software.

The total area of the retropatellar cartilage and the area of wear was measured and used to calculate the percentage of RPC wear, the following were the steps to measure the \% RPC wear: (1) Calibrate and set up the scale 300 pixels $/ \mathrm{mm}$, (2) Draw and then measure the total area of RPC (Freehand), (3) Draw and measure the damaged area of RPC, (4) Calculate the $\%$ of the area that is damaged (Figure 1 A\&B).

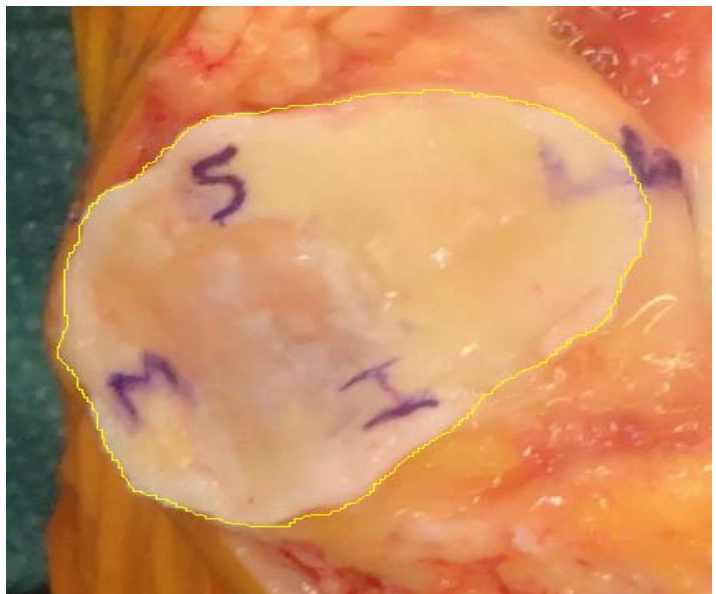

Fig 1A: Measurement of total area of RPC using Image J software

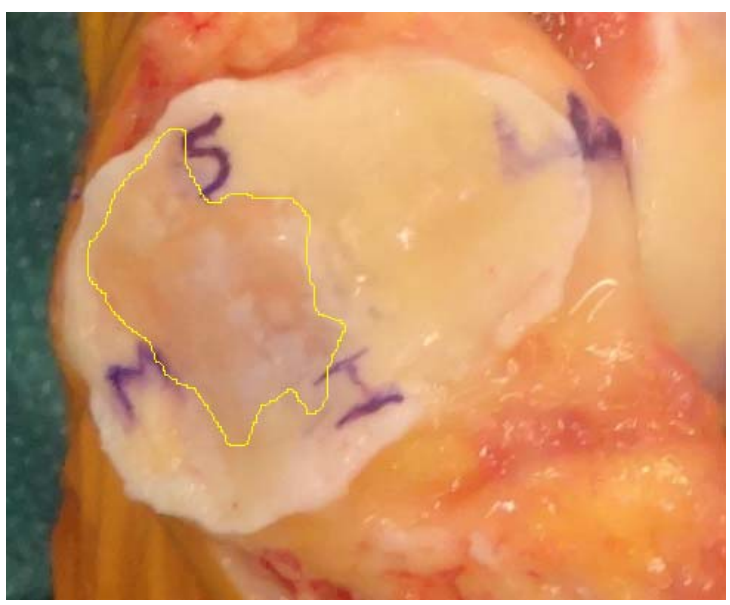

Fig 1B: Measurement of damaged area of RPC using Image J software.

The photographs were then analysed to investigate the patterns of damage i.e. the facets involved in the wear process. The retro patellar cartilage was divided into: medial and lateral facets of the upper, middle and lower thirds of the retro patellar surface (Figure 2). A damaged area was scored a number $(+1)$, and an undamaged area was given a number $(0)$. The following was the standard method used to divide the RPC surface for all photos:

Identify most lateral point of patella.

Draw transverse reference line across patella to most medial point (usually longest line possible).

Find most inferior point of patella (in middle of infrapatellar fat pad).

Draw vertical reference line up from this point perpendicular to the transverse reference line.

Remove transverse reference line and replace with 2 transverse lines perpendicular to the vertical reference line that bisects the line in to equal thirds. 


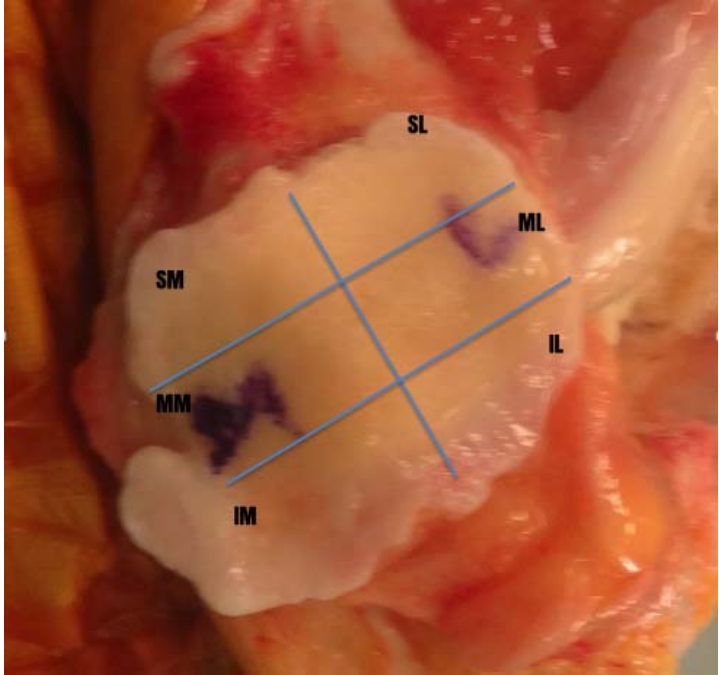

Fig 2: Retro patella Surface-(superior medial-SM, Superior LateralSL, Middle Medial-MM, Middle Lateral-ML, Inferior Medial-IM, Inferior Lateral-IL).

The kneeling ability for all of the patients was recorded before their TKA surgery. The percentages of retro patellar cartilage wear for all the patients were linked with their kneeling ability before the surgery.

Patients were numbered from 1 to 30 (Two Groups-Able and Unable to kneel) and analysed accordingly. SPSS version 21.0 used for statistical analysis; a box and whisker plot was used to assess and compare the link between the kneeling ability before TKA and RPC \% wear in the two groups and ChiSquare Test to evaluate the significance of the relation between the kneeling ability and patterns of wear.

\section{Results}

30 patients participated in the study ( 20 women\& $10 \mathrm{men})$; their mean age was 70.1 yrs $( \pm 9.2) .16$ underwent (Right) TKA and 14 underwent (Left) TKA. (Three of the thirty photographs excluded from the study because 2 of them were not within the standards and one patient missed intraoperative photographing of his patella due to unexpected change of orders of the operating list.

\subsection{Kneeling ability and percentage of RPC wear}

15 patients (cases numbered 1-15, termed group A) were able to kneel, and 15 patients (cases numbered 16-30, termed group B) were unable to kneel. Group A included 6 women and 9 men; they underwent 7 Right TKA and 8 Left TKA; their mean age was 70.6yrs $( \pm 6.0)$, and Group B included 14 women and 1 man; they underwent 9 Right TKA and 6 Left TKA; their mean age was 69.7 yrs $( \pm 11.9)$. RPC wear was observed in both groups. The \% of wears for all RPC images measured 3 times with a week interval and the average of the records in the 1st, 2nd, and 3rd week recorded for statistical analysis.

Mean \% of RPC wears in-group A was (15\%), and in-group B was $(36 \%)$. However, this difference was not significant $(\mathrm{P}$ value $=0.4) .4$ patients with normal RPC unable to kneel, at the same time one patient with $60 \%$ RPC damage still able to kneel.

There was no direct link between the kneeling ability and percentage of RPC wears, as numbers of TKA patients with higher RPC wear were still able to kneel and others with lower RPC \% of wears were unable to kneel, moreover; observed bias noticed in Group B (Figure 3).

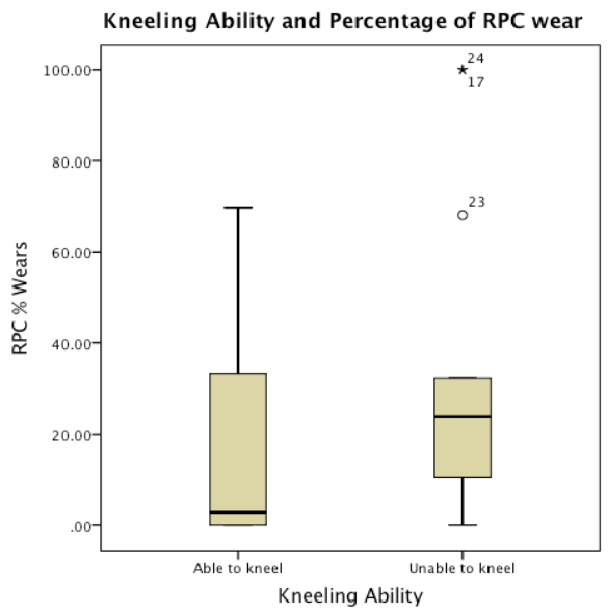

Fig 3: kneeling ability and \% RPC wear (Group A- Patients able to kneel, Group B- Patients unable to kneel.

Intra-Rater and Inter-Rater Reliability statistically assessed by using Intraclass Correlation Coefficient-ICC; the measurements of RPC wear \% performed to all the photographs by myself once a week for 3 weeks resulted in ICC $=0.9$; on the other hand, two orthopaedic surgeons who followed the same standardized steps for the measurements resulted in $\mathrm{ICC}=0.8$. The extent of intra-rater and inter-rater reliability of measurement report according to is conventionally considered to be excellent.

\subsection{Patterns of RPC Wear}

The wear affected the RPC to different extents in both groups (A, those able to kneel and B those, unable to kneel) of TKA patients in both medial and lateral facets (Figure 4\& 5).

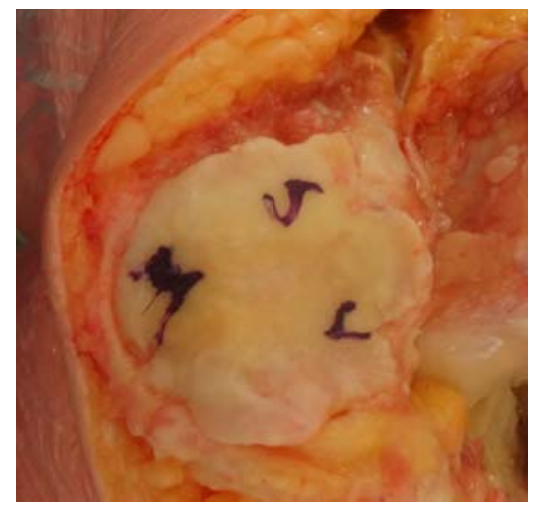

Fig 4: A Photograph (Right RPC) from group A -Able to kneel

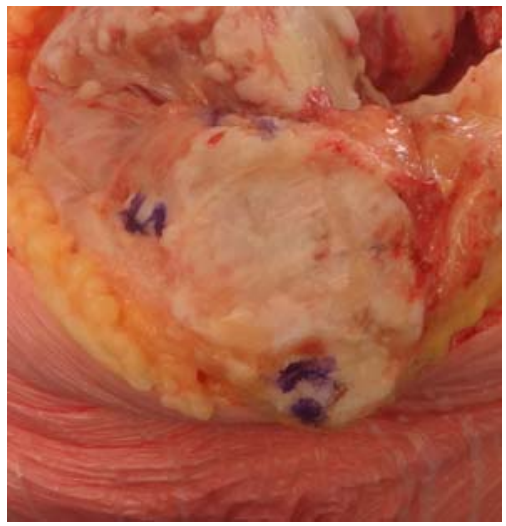

Fig 5: A photograph (Right RPC) from group B - unable to kneel 
The damaged (+1) and undamaged (0) RPC surfaces in both groups A\&B were assessed to determine the patterns of wear and the total number of damaged areas calculated for comparison between the two groups (Figure 6\& 7).

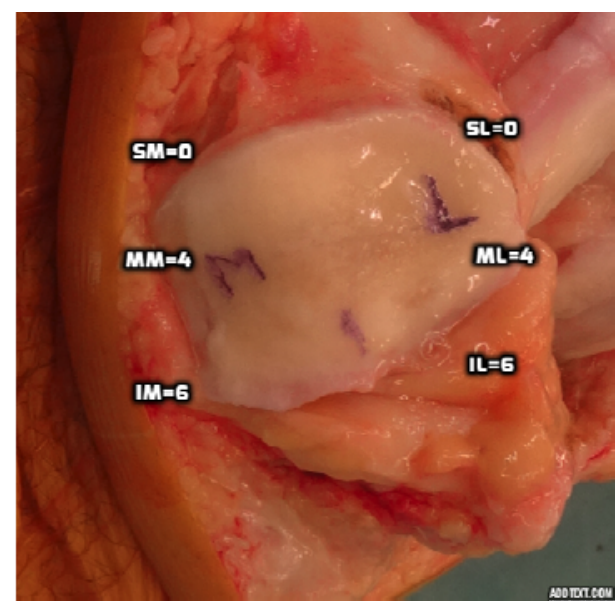

Fig 6: Group A (13 Images)- patients Able to kneel (Frequencies of damages in each surface area-damaged $=+1$, undamaged $=0$ )

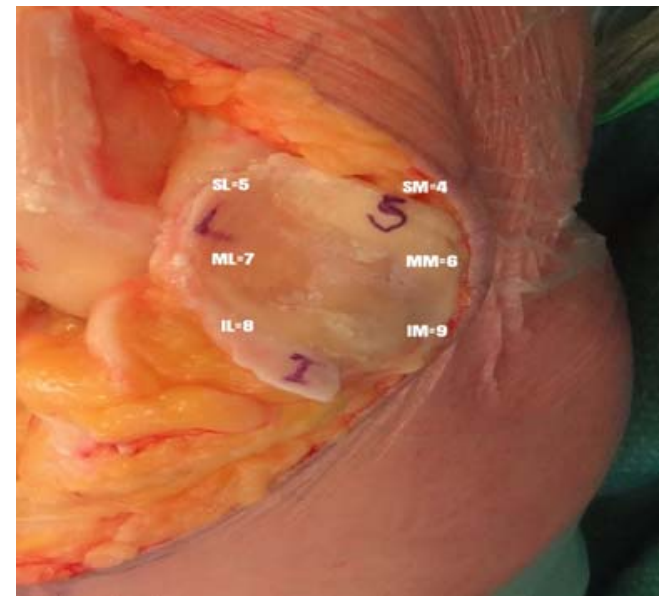

Fig 7: Group B (14 Images)- patients unable to kneel (Frequencies of damages in each surface area-damaged $=+1$, undamaged $=0$ ).

Patients who able to kneel (Group A) had normal RPC superior surfaces with no damages in comparison with patients who unable to kneel (Group B) who had damaged superior surfaces medially and laterally. The middle and inferior surfaces of medial and lateral facets were more affected than superior surfaces in both groups of patients. All the RPC surfaces (Superior, Middle and Inferior) were damaged in Group B patients (Unable to kneel).

There were no distinct patterns of wears both in medial and lateral facets and not directly linked to the ability to kneel.

\section{Discussion}

The purpose of this research study was to determine whether a high \% of RPC wear and the pattern of this wear were related to the kneeling ability before TKA. The most important finding of the present study was that (1) the RPC damaged area does not influence the kneeling ability; and (2) the effect of the amount of RPC damage on the kneeling ability in need of further study especially in cultures with frequent kneeling activities

Few reports in the literature focused on the link between the kneeling function and the retropatellar cartilage wear. The small number of patients in one culture limited this study results. However; the results were important in designing a future larger scale study to include different cultures with different kneeling activities.

The RPC wear area relates to the area where the medial and lateral patellar facets are in contact with the anterior femoral condyle (Gorniak 2009) ${ }^{[12]}$. In the elderly, activities that require a higher knee flexion are performed with either more difficulty or avoided altogether (Gorniak 2009) ${ }^{[12]}$ as a result of decreases in the strength of the knee and hip extensors (Lindle, Metter et al. $1997^{[23]}$, Petrella, Kim et al. 2005) ${ }^{[27]}$.

Abnormal anthropometrics of the patella or distal femoral condyles due to aging or repetitive movement of the knee would alter the joint function and tend to affect the jointspecific wear pattern (Salsich and Perman 2007) ${ }^{[30]}$. Previous studies analysed the RPC wear and patterns of damage. Many of these studies assessed the PF dysfunction but not specifically relate the observed retropatellar cartilage changes to the kneeling ability. Certain studies described the damage in the medial facet (Buff, Jones et al. 1988) ${ }^{[4]}$, (Fulkerson and Shea 1990) ${ }^{[11]}$, (Huberti and Hayes 1984) [18], (Kalichman, Zhang et al. 2007) [20], (Nomura, Inoue et al. 2003) [25], (Potter, Linklater et al. 1998) ${ }^{[28]}$, (Sharma, Song et al. 2001); the lateral facet (Buff, Jones et al. 1988) ${ }^{[4]}$, (Cohen, Mow et al. 2003) [6], (Fulkerson and Shea 1990) [11], (Harilainen, Lindroos et al. 2005), (Huberti and Hayes 1984) [18], (Kalichman, Zhang et al. 2007) ${ }^{[20]}$, (Moro-oka, Matsuda et al. 2002) ${ }^{[24]}$, (Potter, Linklater et al. 1998) ${ }^{[28]}$, (Salsich, Ward et al. 2003) [31], (Sharma, Song et al. 2001), (Thomeé, Augustsson et al. 1999) [35]; the central patella ((Harilainen, Lindroos et al. 2005), (Nomura, Inoue et al. 2003) ${ }^{[25]}$, (Potter, Linklater et al. 1998)) [28]; the medial femoral condyle (Cohen, Mow et al. 2003) [6], (Potter, Linklater et al. 1998) [28] and the lateral femoral condyle (Cohen, Mow et al. 2003) ${ }^{[6]}$, (Nomura, Inoue et al. 2003) ${ }^{[25]}$, (Potter, Linklater et al. 1998) ${ }^{[28]}$. These studies reported that the lateral and medial facets could be damaged with malalignment of the patella. Moreover; the lateral patellar facet is mainly involved with chondromalacia patellae and with patellofemoral compression syndrome. Studies on patellofemoral dysfunction and pain describe increased lateral contact and patellofemoral joint pressure (Cohen, Mow et al. $2003^{[6]}$, Harilainen, Lindroos et

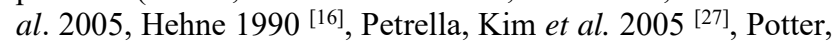

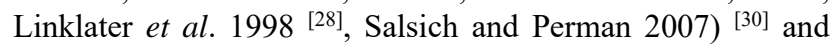
suggests an increase in lateral patellar and lateral femoral articular wear. These studies also indicate that the medial facet shows more damage with aging than the lateral facet (Fulkerson and Shea $1990^{[11]}$, Nomura, Inoue et al. $2003^{[25]}$ ) which is different than the observed RPC damages in this study; the lateral facets and medial facets almost equally affected. The authors of some of these studies described the gender differences in patellofemoral anatomy and mechanics (Besier, Draper et al. 2005, Cicuttini, Wluka et al. 2002, Csintalan, Schulz et al. 2002, Draper, Besier et al. 2006, Grelsamer and Klein 1998, Lindle, Metter et al. $1997^{\text {[23], }}$ Petrella, Kim et al. 2005) ${ }^{[27]}$ but others describe no gender differences (Fulkerson and Shea $1990^{[11]}$, Grelsamer and Klein 1998, Ireland, Willson et al. 2003, Kwak, Colman et al.

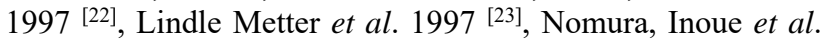

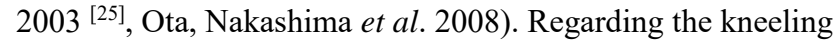
ability in this research study; $70 \%$ of women participated were unable to kneel (14/20) and only one man (10\%) could not kneel before the surgery, furthermore; $90 \%$ of men $(9 / 10)$ and $30 \%$ of women $(6 / 20)$ were able to kneel. The gender effect on kneeling ability is an important observation but 
inconclusive because of a relatively small number of patients involved. According to Gorniak et al. (2009) ${ }^{[12]}$ the pattern of RPC wears was more central and compact in males than in females (gender difference in wear area). This gender difference both in wear area noticed by Gorniak et al. and the observed kneeling ability differences may also relate to males usually having a larger body mass and greater hip and knee muscle strength than female (Petrella, Kim et al. 2005) ${ }^{[27] .}$ Also, this gender difference may relate to hip and knee muscular tightness, restricting the movement and affecting PF joint wear. Because of these and many other factors, and the relatively small sample size, further study is needed to determine if the difference in kneeling ability between males and females is significant and, if so, what particular factors may be involved in this difference.

While many studies analyse the location and severity of wear on the patellar facets for pathological patellofemoral conditions, there is no common RPC wear pattern can be described for all patients. In this study the photographs of the retro patellar cartilage showed that the wear was located in both lateral and medial facets. This current study evaluated the \% of RPC wear and the pattern of wear in 27 patients and the femoral trochlea not assessed; this could be a potential limitation of the study. The results disprove the null hypothesis, postulated that kneeling function limitation in TKA patients is directly linked to the severity and pattern of RPC wear.

A study performed by Gorniak et al. (2009) ${ }^{[12]}$ to detect any common specific-joint wear pattern at the patellofemoral joint in 26 cadavers ( 50 patellae) from older individuals. The study found that there is greater medial patellar facet and odd facet wear than lateral facet wears, suggesting higher medial than lateral patellofemoral joint contact forces. The smaller amount of wear on the anterior aspect of the lateral femoral condyle suggests that contact forces between the lateral facet and anterior aspect of the lateral femoral condyle are low during most of knee flexion. This study agreed with Gorniak's study in that the presence of a common wear pattern at the PFJ would seem unlikely because multiple factors involved such as anatomical variations, tissue pathologies, misalignment, and physical behaviour.

The different joint specific patterns noticed in Gorniaks' study and the results of this study could be considered in further studies of kneeling function and its link with retropatellar cartilage and the retrospective contact area in the anterior femoral condyle.

\section{Conclusion}

The middle and lower thirds of both lateral and medial facets were the most damaged areas whether the patients were able or unable to kneel. Furthermore no direct link between the \% of RPC wear and the patient's kneeling ability. The patterns of retro-patellar cartilage wear were not linked with the kneeling function of TKA patients.

\section{References}

1. Amin S, Goggins J, Niu J, Guermazi A, Grigoryan M, Hunter DJ, Genant HK, Felson DT. Occupation-relate squatting, kneeling, and heavy lifting and the knee joint: a magnetic resonance imaging-based study in men. J Rheumatol, 2008; 35:1645-9.

2. Besier TF, Pal S, Draper CE, Fredericson M, Gold GE, Delp SL, Beaupré GS. The Role of Cartilage Stress in Patellofemoral Pain. Med Sci Sports Exerc, 2015; 47:2416-22.
3. Björkström S, Goldie IF. A study of the arterial supply of the patella in the normal state, in chondromalacia patellae and in osteoarthrosis. Acta Orthop Scand, 1980; 51: 6370 .

4. Buff HU, Jones LC, Hungerford DS. Experimental determination of forces transmitted through the patellofemoral joint. J Biomech, 1988; 21:17-23.

5. Cicuttini F, Wluka A, Wang Y, Stuckey S. The determinants of change in patella cartilage volume in osteoarthritic knees. J Rheumatol, 2002; 29:2615-9.

6. Cohen ZA, Mow VC, Henry JH, Levine WN, Ateshian GA. Templates of the cartilage layers of the patellofemoral joint and their use in the assessment of osteoarthritic cartilage damage. Osteoarthritis Cartilage, 2003; 11:569-79.

7. Csintalan RP, Schulz MM, Woo J, Mcmahon PJ, Lee TQ. Gender differences in patellofemoral joint biomechanics. Clin Orthop Relat Res 2002, 260-9.

8. Draper CE, Besier TF, Gold GE, Fredericson M, Fiene A, Beaupre GS, Delp SL. Is cartilage thickness different in young subjects with and without patellofemoral pain? Osteoarthritis Cartilage, 2006; 14: 931-7.

9. Dye SF. The pathophysiology of patellofemoral pain: a tissue homeostasis perspective. Clin Orthop Relat Res, 2005, 100-10.

10. Froimson MI, Ratcliffe A, Gardner TR, Mow VC. Differences in patellofemoral joint cartilage material properties and their significance to the etiology of cartilage surface fibrillation. Osteoarthritis Cartilage, 1997; 5:377-86.

11. Fulkerson JP, Shea KP. Disorders of patellofemoral alignment. J Bone Joint Surg Am, 1990; 72:1424-9.

12. Gorniak GC. Patterns of patellofemoral articular cartilage wear in cadavers. J Orthop Sports Phys Ther, 2009; 39:675-83.

13. Grelsamer RP, Klein JR. The biomechanics of the patellofemoral joint. J Orthop Sports Phys Ther, 1998; 28: 286-98.

14. Grelsamer RP, Proctor CS, Bazos AN. Evaluation of patellar shape in the sagittal plane. A clinical analysis. Am J Sports Med, 1994; 22:61-6.

15. Harilainen A, Lindroos M, Sandelin J, Tallroth K, Kujala UM. Patellofemoral relationships and cartilage breakdown. Knee Surg Sports Traumatol Arthrosc, 2005; 13: 142-4.

16. Hehne HJ. Biomechanics of the patellofemoral joint and its clinical relevance. Clin Orthop Relat Res 1990, 73-85.

17. Horng A, Raya J, Zscharn M, König L, Notohamiprodjo M, Pietschmann M, Hoehne-Hückstädt U, Hermanns I, Glitsch U, Ellegast R, Hering K, Reiser M, Glaser C. [Locoregional deformation pattern of the patellar cartilage after different loading types - high-resolution 3D-MRI volumetry at $3 \mathrm{~T}$ in-vivo]. Rofo, 2011; 183:43240.

18. Huberti HH, Hayes WC. Patellofemoral contact pressures. The influence of q-angle and tendofemoral contact. J Bone Joint Surg Am, 1984; 66:715-24.

19. Ireland ML, Willson JD, Ballantyne BT, Davis IM. Hip strength in females with and without patellofemoral pain. J Orthop Sports Phys Ther, 2003; 33:671-6.

20. Kalichman L, Zhang Y, Niu J, Goggins J, Gale D, Felson DT, Hunter D. The association between patellar alignment and patellofemoral joint osteoarthritis features-an MRI study. Rheumatology (Oxford), 2007; 46:13038. 
21. Kayler DE, Lyttle D. Surgical interruption of patellar blood supply by total knee arthroplasty. Clin Orthop Relat Res 1988, 221-7.

22. Kwak SD, Colman WW, Ateshian GA, Grelsamer RP, Henry JH, Mow VC. Anatomy of the human patellofemoral joint articular cartilage: surface curvature analysis. J Orthop Res, 1997; 15:468-72.

23. Lindle RS, Metter EJ, Lynch NA, Fleg JL, Fozard JL, Tobin J, Roy TA, Hurley BF. Age and gender comparisons of muscle strength in 654 women and men aged 20-93 yr. J Appl Physiol 1985-1997; 83:1581-7.

24. Moro-Oka T, Matsuda S, Miura H, Nagamine R, Urabe $\mathrm{K}$, Kawano T, Higaki H, Iwamoto Y. Patellar tracking and patellofemoral geometry in deep knee flexion. Clin Orthop Relat Res, 2002, 161-8.

25. Nomura E, Inoue $M$, Kurimura $M$. Chondral and osteochondral injuries associated with acute patellar dislocation. Arthroscopy, 2003; 19:717-21.

26. Ota S, Nakashima T, Morisaka A, Ida K, Kawamura M. Comparison of patellar mobility in female adults with and without patellofemoral pain. J Orthop Sports Phys Ther, 2008; 38:396-402.

27. Petrella JK, Kim JS, Tuggle SC, Hall SR, Bamman MM. Age differences in knee extension power, contractile velocity, and fatigability. J Appl Physiol 1985-2005; 98:211-20.

28. Potter HG, Linklater JM, Allen AA, Hannafin JA, Haas SB. Magnetic resonance imaging of articular cartilage in the knee. An evaluation with use of fast-spin-echo imaging. J Bone Joint Surg Am, 1998; 80: 1276-84.

29. Rhee SJ, Haddad FS. Patellofemoral joint in total knee replacement, Current Orthopaedics. 2008; 22(2): 75-154.

30. Salsich GB, Perman WH. Patellofemoral joint contact area is influenced by tibiofemoral rotation alignment in individuals who have patellofemoral pain. J Orthop Sports Phys Ther, 2007; 37:521-8.

31. Salsich GB, Ward SR, Terk MR, Powers CM. In vivo assessment of patellofemoral joint contact area in individuals who are pain free. Clin Orthop Relat Res, 2003, 277-84.

32. Dunlop DD. The role of knee alignment in disease progression and functional decline in knee osteoarthritis. JAMA, 2001; 286:188-95.

33. Staeubli HU, Bollmann C, Kreutz R, Becker W, Rauschning W. Quantification of intact quadriceps tendon, quadriceps tendon insertion, and suprapatellar fat pad: MR arthrography, anatomy, and cryosections in the sagittal plane. AJR Am J Roentgenol, 1999; 173:691-8.

34. Teichtahl AJ, Wluka AE, Wang Y, Urquhart DM, Hanna FS, Berry PA, Jones G, Cicuttini FM. Occupational activity is associated with knee cartilage morphology in females. Maturitas, 2010; 66:72-6.

35. Thomeé R, Augustsson J, Karlsson J. Patellofemoral pain syndrome: a review of current issues. Sports Med, 1999; 28:245-62.

36. Van Der Heijden RA, Oei EH, Bron EE, Van Tiel J, Van Veldhoven PL, Klein S, Verhaar JA, Krestin GP, BiermaZeinstra SM, Van Middelkoop M. No Difference on Quantitative Magnetic Resonance Imaging in Patellofemoral Cartilage Composition between Patients with Patellofemoral Pain and Healthy Controls. Am J Sports Med, 2016; 44:1172-8.

37. Yoshioka Y, SIU D, COOKE TD. The anatomy and functional axes of the femur. J Bone Joint Surg Am, 1987; 69:873-80. 